\title{
ON INFINITE-DIMENSIONAL MANIFOLD TRIPLES
}

\author{
KATSURO SAKAI AND RAYMOND Y. WONG
}

\begin{abstract}
Let $Q$ denote the Hilbert cube $[-1,1]^{\omega}, s=(-1,1)^{\omega}$ the pseudo-interior of $Q, \Sigma=\left\{\left(x_{i}\right) \in s|\sup | x_{i} \mid<1\right\}$ and $\sigma=\left\{\left(x_{i}\right) \in s \mid x_{i}=0\right.$ except for finitely many $i\}$. A triple $(X, M, N)$ of separable metrizable spaces is called a $(Q, \Sigma, \sigma)$ - (or $(s, \Sigma, \sigma)$-)manifold triple if it is locally homeomorphic to $(Q, \Sigma, \sigma)$ (or $(s, \Sigma, \sigma))$. In this paper, we study such manifold triples and give some characterizations.
\end{abstract}

\section{INTRODUCTION}

Throughout this paper, spaces are separable and metrizable. A manifold modeled on a given space $E$ is called an $E$-manifold. For a given pair $(E, F)$ (or triple $(E, F, G))$ of spaces, an $(E, f)$-manifold pair (or $(E, F, G)$-manifold triple) is a pair $(X, M)$ (or triple $(X, M, N))$ of spaces such that there is an open cover $\mathscr{U}$ of $X$ and open embeddings $\phi_{U}: U \rightarrow E, U \in \mathscr{U}$, such that $\phi_{U}(M \cap U)=F \cap \phi_{U}(U)$ (and respectively $\phi_{U}(N \cap U)=G \cap \phi_{U}(U)$ ). Let $Q$ denote the Hilbert cube $[-1,1]^{\omega}, s=(-1,1)^{\omega}$ (the pseudo-interior of $Q$ ), $\Sigma=\left\{\left(x_{i}\right) \in s|\sup | x_{i} \mid<1\right\}=\bigcup_{n=1}^{\infty}\left[-1+n^{-1}, 1-n^{-1}\right]^{\omega}$ and $\sigma=\left\{\left(x_{i}\right) \in s \mid x_{i}=0\right.$ except for finitely many $i\}$. In this paper we study $(Q, \Sigma, \sigma)-($ or $(s, \Sigma, \sigma)-)$ manifold triples.

A closed subset $A$ of a space $X$ is called a $Z$-set in $X$ if for each map $f: Q \rightarrow X$ and each $\varepsilon>0$, there is a map $g: Q \rightarrow X \backslash A$ with $D(f, g)<\varepsilon$. A subset $M$ of $X$ is called a cap (or f.d. cap) set for $X$ if $M=\bigcup_{n \in \mathbf{N}} M_{n}$, where $M_{1} \subset M_{2} \subset \cdots$ is a tower of (resp. finite-dimensional) compact $Z$-sets in $X$ satisfying the following conditions:

(*) For each (finite-dimensional) compact set $A$ in $X$, each $\varepsilon>0$ and each $m \in \mathbf{N}$, there is an integer $n \geq m$ and an embedding $h: A \rightarrow M_{n}$ such that $h \mid A \cap M_{m}=$ id and $d(h$, id $)<\varepsilon$,

where $d$ is a metric for $X\left[\mathrm{An}_{3}\right.$ and $\left.\mathrm{Ch}_{1}\right]$. We call $M$ a strong (f.d.) cap set for $X$ if in the above definition $M=\bigcup_{n \in \mathbf{N}} M_{n}$ satisfies the following stronger condition:

Received by the editors June 10, 1988 .

1980 Mathematics Subject Classification (1985 Revision). Primary 57N20.

Key words and phrases. $(Q, \Sigma, \sigma)$-manifold triple, $(s, \Sigma, \sigma)$-manifold triple, (f.d.) cap pair, cap manifold pair.

This work was done while the first author was visiting the University of California at Santa Barbara. 
(*) For each ( finite-dimensional) compact $Z$-set $A$ in $X$, each open cover $\mathscr{U}$ of $X$ and each $m \in \mathbf{N}$, there is an $n \geq m$ and a homeomorphism $h: X \rightarrow X$ such that $h \mid M_{m}=\mathrm{id}, h(A) \subset M_{n}$ and $h$ is $\mathscr{U}$-close to id.

A strong (f.d.) cap set is called a $\mathscr{Z}$-skeletoid (or $\mathscr{E} \mathscr{Z}$-skeletoid) in $\left[\mathrm{BP}_{1}\right]$, where $\mathscr{Z}$ (or $\mathscr{E} \mathscr{Z}$ ) is the collection of (finite-dimensional) compact $Z$-sets in $X$. In case $X$ is complete-metrizable, strong (f.d.) cap sets are topologically unique, that is, if $M$ and $N$ are strong cap sets (or strong f.d. cap sets) for $X$ then there is a homeomorphism $h: X \rightarrow X$ such that $h(M)=N\left(\left[\mathrm{BP}_{2}\right.\right.$, Chaper IV, Theorem 2.1]). In case $X$ is a $Q$ - (or $s$-)manifold, (f.d.) cap sets for $X$ are strong (f.d.) cap sets [ $\mathrm{BP}_{2}$, Chapter IV, Proposition 4.1]. Note that $\Sigma$ is a cap set and $\sigma$ is an f.d. cap set for both $Q$ and $s$. A pair $(X, M)$ is a $(Q, \Sigma)$ - (or $(s, \Sigma)$-)manifold pair if and only if $X$ is a $Q$ - (or $s$-)manifold and $M$ is a cap set for $X$. A pair $(X, N)$ is a $(Q, \sigma)$ - (or $s, \sigma)$-)manifold pair if and only if $X$ is a $Q$-(or $s$-)manifold and $N$ is an f.d. cap set for $X\left[\mathrm{Ch}_{1}\right]$.

It is well known that $s$ is homeomorphic to $(\cong)$ the separable Hilbert space $l_{2}\left[\mathrm{An}_{1}\right]$. Let $l_{2}^{Q}$ be the linear span of the Hilbert cube $\prod_{i \in \mathrm{N}}\left[-i^{-1}, i^{-1}\right]$ in $1_{2}$, i.e., $l_{2}^{Q}=\left\{\left(x_{i}\right) \in l_{2}|\sup | i x_{i} \mid<\infty\right\}$, and $l_{2}^{f}$ the linear span of the usual orthonormal basis, i.e., $l_{2}^{f}=\left\{\left(x_{i}\right) \in l_{2} \mid x_{i}=0\right.$ except for finitely many $\left.i\right\}$. Then $l_{2}^{Q}$ is a cap set and $l_{2}^{f}$ is an f.d. cap set for $l_{2}$; hence $\left(l_{2}, l_{2}^{Q}\right) \cong(s, \Sigma)$ and $\left(l_{2}, l_{2}^{f}\right) \cong(s, \sigma)$. It is not clear whether $\left(l_{2}, l_{2}^{Q}, l_{2}^{f}\right) \cong(s, \Sigma, \sigma)$, or whether any one of the following triples homeomorphic to $(Q, \Sigma, \sigma)$ or $(s, \Sigma, \sigma)$ :

$$
\left(Q, Q \backslash s, Q_{f} \backslash s\right), \quad\left(Q, \Sigma^{\prime}, \sigma\right), \quad\left(s, \Sigma^{\prime}, \sigma\right), \quad\left(I^{\omega}, I^{\omega} \backslash \stackrel{\circ}{I}^{\omega}, I_{f}^{\omega}\right),
$$

where $Q_{f}=\left\{\left(x_{i}\right) \in Q \mid x_{i}=0\right.$ except for finitely many $\left.i\right\}, \Sigma^{\prime}=\left\{\left(x_{i}\right) \in s|| x_{i} \mid<\right.$ $\frac{1}{2}$ except for finitely many $\left.i\right\}, I=[0,1], \stackrel{\circ}{I}=(0,1)$ and $I_{f}^{\omega}=I^{\omega} \cap Q_{f}$. In general, we can ask whether $(X, M, N)$ is a $(Q, \Sigma, \sigma)$ - (or $(s, \Sigma, \sigma)$-)manifold triple when $X$ is a $Q$ - (or $s$-)manifold, $M$ is a cap set and $N$ is an f.d. cap set for $X$ such that $N \subset M$. This question relates to a conjecture on function spaces as follows: The triple

$$
(C(X, Y), \quad L I P(X, Y), \quad P L(X, Y))
$$

is an $(s, \Sigma, \sigma)$-manifold triple if $X$ and $Y$ are polyhedra in $\mathbb{R}^{n}, X$ is compact, $\operatorname{dim} X \neq 0$ and $Y$ has no isolated point (or $Y$ is open in $\mathbb{R}^{n}$ ), where $C(X, Y)$ is the space of maps from $X$ to $Y, L I P(X, Y)$ the subspace of $C(X, Y)$ consisting of all Lipschitz maps and $P L(X, Y)$ the subspace of $C(X, Y)$ consisting of all piecewise linear maps (cf. $\left.[\mathrm{Ge}],\left[\mathrm{Sa}_{3}\right],[\mathrm{SW}]\right)$.

In $\S 2$, we give a characterization of $(Q, \Sigma, \sigma)$ - $($ or $(s, \Sigma, \sigma))$-manifold triples. Using this characterization, we can easily show, among other results, that $\left(l_{2}, l_{2}^{Q}, l_{2}^{f}\right) \cong(s, \Sigma, \sigma),\left(Q, Q \backslash s, Q_{f} \backslash s\right) \cong(Q, \Sigma, s)$, etc. This section contains our principal results on manifold triples.

In $\S 3$, we establish a relationship between $(\Sigma, \sigma)$-manifold pairs and $\Sigma \times s$ manifolds. In fact, we show that $\Sigma \backslash \sigma \cong \Sigma \times s$; for any $(\Sigma, \sigma)$-manifold pair 
$(M, N), M \backslash N$ is a $\Sigma \times s$-manifold and each $\Sigma \times s$-manifold is homeomorphic to $M \backslash N$ for some $(\Sigma, g)$-manifold pair $(M, N)$. Using the fact $\Sigma \backslash \sigma \cong \Sigma \times s$, we can easily construct a cap set $M$ and an f.d. cap set $N$ for both $Q$ and $s$ so that $N \subset M$ but $(Q, M, N) \not(Q, \Sigma, \sigma)$ and $(s, M, N) \not(s, \Sigma, \sigma)$.

Example. Let $N=\left\{\left(x_{i}\right) \in s \mid x_{i}=\frac{2}{3}\right.$ except for finitely many $\left.i\right\}$ and $M=$ $\Sigma^{\prime} \cup N$. Then $N$ is clearly an f.d. cap set for both $Q$ and $s$ and $M$ is a cap set for both $Q$ and $s$ ([ $\mathrm{Ch}_{1}$, Theorem 6.6]). Hence $M \backslash N=\Sigma^{\prime} \cong \Sigma \not \Sigma \times s \cong \Sigma \backslash \sigma$ since $\Sigma \times s$ is not $\sigma$-compact. Therefore $(M, N) \not \equiv(\Sigma, \sigma)$, which implies $(Q, M, N) \not(Q, \Sigma, \sigma)$ and $(s, M, N) \not(s, \Sigma, \sigma)$.

Applying the result of $\S 2$, we give in $\S 3$ a characterization of $(\Sigma, \sigma)$-manifold pairs and show that $(X, M, N)$ is a $(Q, \Sigma, \sigma)$ - (or $(s, \Sigma, \sigma)$-)manifold triple if and only if $(X, M)$ is a $(Q, \Sigma)$ - (or $(s, \Sigma)$-)manifold pair (or $(X, N)$ is a $(Q, \sigma)$ - (or $(s, \sigma)$-)manifold pair) and $(M, N)$ is a $(\Sigma, \sigma)$-manifold pair.

Notations and terminology. A metric for a space $X$ is denoted by $d$. for $A \subset X$ and $x \in X$, let $d(x, A)=\inf \{d(x, a) \mid a \in A\}$. For maps $f, g: Y \rightarrow X$, let $d(f, g)=\sup \{d(f(y), g(y)) \mid y \in Y\}$. For $A \subset X$ and a collection $\mathscr{B}$ of subsets of $X$, let $\operatorname{st}(A, \mathscr{B})=\bigcup\{B \in \mathscr{B} \mid A \cap B \neq 0\}$. Let $\mathscr{A}$ and $\mathscr{B}$ be collections of subsets of $X$. Define st $(\mathscr{A}, \mathscr{B})=\{\operatorname{st}(A, B) \mid A \in \mathscr{A}\}$. For $n \in$ $\mathbf{N}$, let $\mathbf{s t}^{n}(\mathscr{B})=\mathbf{s t}\left(\mathbf{s t}^{n-1}(\mathscr{B}), \mathscr{B}\right)$, where $\mathbf{s t}^{0}(\mathscr{B})=\mathscr{B}$. We denote $\mathscr{A}<\mathscr{B}$ if each $A \in \mathscr{A}$ is contained is some $B \in \mathscr{B}$. Maps $f, g: Y \rightarrow X$ are said to be $\mathscr{B}$-close if $\{\{f(y), g(y)\} \mid y \in Y\}<\mathscr{B}$. A homotopy $h: Y \times I \rightarrow X$ is called a $\mathscr{B}$-homotopy if $\{\{h(\{y\} \times I)\} \mid y \in Y\}<\mathscr{B}$. Then we say that $h_{0}=h \mid Y \times\{0\}$ is $\mathscr{B}$-homotopic to $h_{1}=h \mid Y \times\{1\}$ and denote $h_{0} \stackrel{\mathscr{B}}{\simeq} h_{1}$. Let $\mathscr{U}$ be an open cover of $X$. A map $f: Y \rightarrow X$ is called a $\mathscr{U}$-homotopy equivalence if there is a map $g: X \rightarrow Y$ called a $\mathscr{U}$-homotopy inverse of $f$ such that $f g \stackrel{\mathscr{U}}{\simeq} \mathrm{id}_{x}$ and $g f^{f^{-1}(\mathscr{U})} \simeq \mathrm{id}_{y}$. A fine homotopy equivalence $f: Y \rightarrow X$ is a $\mathscr{U}$-homotopy equivalence for any open cover $\mathscr{U}$ of $X$. Let $A$ be a closed set in $X$. An open cover $\mathscr{V}$ of $X \backslash A$ is said to be normal relative to $A$ provided any homeomorphism $h: X \backslash A \rightarrow X \backslash A$ of $X \backslash A$ onto itself which is $\mathscr{V}$-close to id can be extended to a homeomorphism $h: X \rightarrow X$ of $X$ onto itself by $\tilde{h} \mid A=$ id .

\section{PAir Versions OF (F.D.) CAP SETS}

Let $X$ be a space and $y \subset X$, A strong (f.d.) cap set $M$ for $X$ is called a strong (f.d.) cap set for $(X, Y)$ if $h$ in $(*)_{s}$ above satisfies $h(Y)=Y$. Let $H(X)$ be the group of homeomorphisms of $X$ onto itself and $H(X, Y)$ the subgroup of $H(X)$ consisting of all $h \in H(X)$ satisfying $h(Y)=Y$. A strong (f.d.) cap set for $(X, Y)$ is called an $H(X, Y)$ - $\mathscr{Z}$-skeletoid (or $H(X, Y)$ skeletoid) in $\left[\mathrm{BP}_{2}\right]$. In general $H(X, Y)$ is not closed in $H(X)$ in the topology of uniform convergence with respect to any metric for $X$. Hence the following lemma does not follow immediately from [ $\mathrm{BP}_{2}$, Chapter IV, Proposition 2.2]. 
1.1. Lemma. Let $X$ be complete-metrizable and $Y \subset X$. Then for any two strong (f.d.) cap sets $M$ and $N$ for $(X, Y)$ and any open cover $\mathscr{U}$ of $X$, there is a homeomorphism $h:(X, M, Y) \rightarrow(X, N, Y)$ which is $\mathscr{U}$-close to id.

Proof. A proof can be given following the same procedure as in $\left[\mathrm{Ch}_{1}\right.$, Lemma 4.3] and will be omitted.

Let $(M, N)$ be a pair of subsets of a space $X$. We call $(M, N)$ a (cap, f.d. cap)-pair for $X$ if $M=\bigcup_{n=1}^{\infty} M_{n}$, where $M_{1} \subset M_{2} \subset \cdots$ is a tower of compact $Q$-manifolds satisfying $(*)$ above and the following: $M_{n}$.

(*) Each $M_{n}$ is a $Z$-set for $M_{n+1}$ and each $M_{n} \cap N$ is an f.d. cap set for

Remark. It follows that $N$ is an f.d. cap set for $X$.

1.2. Lemma. Let $(M, N)$ be a (cap, f.d. cap)-pair for a $Q$ - (or s-)manifold $X$. Then $M$ is a strong cap set for $(X, N)$.

Proof. Write $M=\bigcup_{n \in \mathbf{N}} M_{n}$ as in the definition of (cap, f.d. cap)-pair. Let $\mathscr{U}$ be an open cover of $X$ and $A$ a compact $Z$-set in $X$. From (*), there is an $n \geq m$ and an embedding $f: A \rightarrow M_{n}$ such that $f \mid A \cap M_{m}=$ id and $f$ is $\mathscr{U}$-homotopic to id. By [ $\mathrm{Ch}_{1}$, Lemma 5.4, Theorems 6.7 and 6.6],

$$
L=\left(M_{n+1} \cap N\right) \backslash f\left(A \cap M_{m}\right)
$$

and

$$
L^{\prime}=\left(\left(\left(M_{n+1} \cap N\right) \backslash f(A)\right) \cup f(A \cap N)\right) \backslash f\left(A \cap M_{m}\right)
$$

are f.d. cap sets for $M_{n+1} \backslash f\left(A \cap M_{m}\right)$. Let $\mathscr{V}$ be an open cover of $M_{n+1} \backslash f\left(A \cap M_{m}\right)$ such that $\mathscr{V}<\mathscr{U}$ and $\mathscr{V}$ is normal relative to $f\left(A \cap M_{m}\right)$. By [ $\mathrm{Ch}_{1}$, Theorem 6.2], we have a homeomorphism of pair

$$
g^{\prime}:\left(M_{n+1} \backslash f\left(A \cap M_{m}\right), L^{\prime}\right) \rightarrow\left(M_{n+1} \backslash f\left(A \cap M_{m}\right), L\right)
$$

which is $\mathscr{V}$-homotopic to id. Then $g^{\prime}$ extends to a homeomorphism $g: M_{n+1}$ $\rightarrow M_{n+1}$ with $g \mid f\left(A \cap M_{m}\right)=$ id, and $g$ is $\mathscr{U}$-homotopic to id. Observe that $g f(A \cap N) \subset N$ and $g f(A \backslash N) \subset M_{n+1} \backslash N$; that is, $g f(A) \cap N=g f(A \cap N)$. Since $g f: A \rightarrow X$ is a $Z$-embedding which is st $\mathscr{U}$-homotopic to id, $g f$ extends to a homeomorphism $h: X \rightarrow X$ which is st $^{2} \mathscr{U}$-isotopic to id by the Homeomorphism Extension Theorem [AC]. By [ $\mathrm{Ch}_{1}$, Lemma 5.4], $N \backslash h(A)$ and $h(N \backslash A)=h(N) \backslash h(A)$ are f.d. cap set for $X \backslash h(A)$. Similarly, there is a homeomorphism $k: X \rightarrow X$ such that $k \mid h(A)=\mathrm{id}, k h(N \backslash A)=N \backslash h(A)$ and $k$ is $\mathscr{U}$-close to id. Since $h(A \cap N)=h(A) \cap N$, we have $k h(N)=N$. Thus we have a homeomorphism $k h: X \rightarrow X$ such that $k h(N)=N, k h \mid A \cap M_{m}=$ $g f \mid A \cap M_{m}=$ id, $k h(A)=g f(A) \subset g\left(M_{n}\right) \subset M_{n+1}$ and $k h$ is st $^{3} \mathscr{U}$-close to id.

For any countable locally finite simplicial complex $K,|K| \times Q$ is a $Q$ manifold and $|K| \times s$ is an $s$-manifold [We]. As easily observed, $(\Sigma, \sigma)$ is 
$a$ (cap, f.d. cap)-pair for both $Q$ and $s$. It is straightforward to verify the following:

1.3. Lemma. For any countable locally finite simplicial complex $K,(|K| \times$ $\Sigma,|K| \times \sigma)$ is a (cap, f.d. cap)-pair for both $|K| \times Q$ and $|K| \times s$.

It is also easy to verify that various versions of $(\Sigma, \sigma)$ mentioned in the introduction are (cap, f.d. cap)-pairs. The following is rather useful in the proof of our characterization.

1.4. Lemma. Let $(M, N)$ be a (cap, f.d. cap)-pair for a $Q$ - (or s-) manifold $X$ and $U$ an open set in $X$. Then $(M \cap U, N \cap U)$ is a (cap, f.d. cap)-pair for $U$.

Proof. Let $M=\bigcup_{n \in \mathbf{N}} M_{n}$ be as in the definition and for each $n \in \mathbf{N}$, let $A_{n}=\{x \in U \mid d(x, X \backslash U) \leq 1 / n\}$. Without loss of generality, we may assume that $M_{1} \cap A_{1} \neq \varnothing$. By [ $\left.\mathrm{Ch}_{2}\right], M_{1} \cap U \cong|K| \times Q$ for some countable locally finite simplical complex $K$. Hence, $|K| \times \sigma$ is an f.d. cap set for $|K| \times Q$. On the other hand, $M_{1} \cap N \cap U$ is an f.d. cap set for $M_{1} \cap U$ by [ $\mathrm{Ch}_{2}$, Lemma 5.4]. By [ $\mathrm{Ch}_{1}$, Theorem 6.2], we have a homeomorphism

$$
h:\left(M_{1} \cap U, M_{1} \cap N \cap U\right) \rightarrow(|K| \times Q,|K| \times \sigma) .
$$

Since $M_{1} \cap A_{1}$ is compact, $h\left(M_{1} \cap A_{1}\right) \subset|L| \times Q$ for some finite subcomplex $L$ of $K$. Let $M_{1}^{*}=h^{-1}(|L| \times Q)$. Then $M_{1}^{*}$ is a compact $Q$-manifold with $M_{1} \cap A_{1} \subset M_{1}^{*} \subset M_{1} \cap U$ and $M_{1}^{*} \cap N \cap U=h^{-1}(|L| \times \sigma)$ an f.d. cap set. Using this process inductively, we obtain compact $Q$-manifolds $M_{g n}^{*}, n \in \mathbf{N}$, such that $M_{n-1}^{*} \cup\left(M_{n} \cap A_{n}\right) \subset M_{n}^{*} \subset M_{n} \cap U$ and $M_{n}^{*} \cap N \cap U$ is an f.d. cap set for $M_{n}^{*}$. Then $M \cap U=\bigcup_{n \in \mathbf{N}} M_{n}^{*}$ satisfies (*) and (**) relative $U$ and $N \cap U$.

A subset $M$ of a space $X$ said to be map-dense in $X$ if for each compact set $A$ in $X$ and each $\varepsilon>0$, there is a map $f: A \rightarrow M$ such that $d(f$, id $)<\varepsilon$.

1.5. Lemma. Let $(M, N)$ be a pair of subsets of a space $X$ such that $M$ is an ANR. Then $(M, N)$ is a (cap, f.d. cap)-pair for $X$ if and only if $M$ is mapdense in $X$ and $M=\bigcup_{n \in \mathbf{N}} M_{n}$, where $M_{1} \cap M_{2} \subset \cdots$ is a tower of compact $Q$-manifolds satisfying $(* *)$ and the following:

$(*)^{\prime}$ For each compact set $A$ in $M$, each $\varepsilon>0$ and each $m \in \mathbf{N}$, there is an $n \geq m$ and a map $f: A \rightarrow M_{n}$ with $d(f$, id $)<\varepsilon$, and $f\left(A \cap M_{m}\right) \subset M_{m}$. Proof. The "only if" part is trivial. By [ $\mathrm{Sa}_{2}$, Proposition 2.1] (cf. [ $\mathrm{Sa}_{1}$, Lemma $1]$ ), we can verify that $\left\{M_{n}\right\}_{n \in \mathbf{N}}$ satisfies (*).

Since a cap set for a $Q$ - (or $s$-)manifold is a $\Sigma$-manifold, we have the following.

1.6. Corollary. Let $(M, N)$ be a (cap, f.d. cap)-pair for a $Q$ - (or $S$-) manifold $X$. Then for any homeomorphism $f: M \rightarrow M$ of $M$ onto itself and $M \subset Y \subset$ $X,(M, f(N))$ is a (cap, f.d. cap)-pair for $Y$. 
2. Characterization of $(Q, \Sigma, \sigma)$ - (OR $(s, \Sigma, \sigma)$ ))MANifold triples

Here we give a characterization of $(Q, \Sigma, \sigma)$ - (or $(s, \Sigma, \sigma)$-)manifolds.

2.1. Theorem. Let $X$ be a $Q$ - (or $s$-) manifold and $N \subset M \subset X$. Then the following are equivalent:

(i) $(X, M, N)$ is a $(Q, \Sigma, \sigma)$ - (or $(s, \Sigma, \sigma)$-) manifold triple ;

(ii) $(M, N)$ is a (cap, f.d. cap)-pair for $X$;

(iii) $M$ is a strong cap set for $(X, N)$ and $N$ is an f.d. cap set for $X$.

Proof. The implication (ii) $\Rightarrow$ (iii) is Lemma 1.2 and (iii) $\Rightarrow$ (ii) follows from Lemmas 1.3, 1.2 and 1.1. We shall prove the equivalence of (i) and (ii).

(ii) $\Rightarrow$ (i): Each $x \in X$ has an open neighborhood $U$ with an open embedding $g: U \rightarrow Q$ (or $g: U \rightarrow s$ ). By Lemma 1.4, $(M \cap U, N \cap U)$ and $(\Sigma \cap g(U), \sigma \cap g(U))$ are (cap, f.d. cap)-pairs for $U$ and $g(U)$, respectively. Then by Lemmas 1.2 and 1.1, there is a homeomorphism

$$
\phi:(U, M \cap U, N \cap U) \rightarrow(g(U), \Sigma \cap g(U), \sigma \cap g(U)) .
$$

Therefore $(X, M, N)$ is a $(Q, \Sigma, \sigma)$ - (or $(s, \Sigma, \sigma)$-)manifold triple.

(i) $\Rightarrow$ (ii): By $\left[\mathrm{Ch}_{2}\right] X \cong|K| \times Q$ (or $|K| \times s$ ) for some countable locally finite simplicial complex $K$. Since $(|K| \times \Sigma,|K| \times \sigma)$ is a (cap, f.d. cap)-pair for $|K| \times Q$ (or $|K| \times s)$ by Lemma 1.3, we have a (cap, f.d. cap)-pair $\left(M^{\prime}, N^{\prime}\right.$ ) for $X$. From the definition of manifold triples and Lemma 1.4, $X$ has a countable star-finite open cover $\mathscr{U}$ such that for each $U \in \mathscr{U},(M \cap U, N \cap U)$ is a (cap, f.d. cap)-pair for $U$. By [AHW] there is an ordering $\left\{U_{i}\right\}_{i \in \mathbf{N}}$ of $\mathscr{U}$ and a sequence $n_{1} \leq n_{2} \leq \cdots$ in $\mathbf{N}$ such that for any $f_{i} \in H(X), i \in \mathbf{N}$, with $f_{i} \mid X \backslash U_{i}=\mathrm{id},\left\{f_{i} \circ \cdots \circ f_{1}\right\}_{i \in \mathbf{N}}$ converges pointwise to an $f \in H(X)$ with $f\left|U_{i}=f_{n_{1}} \circ \cdots \circ f_{n_{i}}\right| U_{i}$. Note that $\left(M^{\prime} \cap U_{1}, N^{\prime} \cap U_{1}\right)$ is a (cap, f.d. cap)-pair for $U_{1}$ by Lemma 1.4. Using Lemmas 1.2 and 1.1, we have a homeomorphism

$$
h_{1}:\left(U_{1}, M^{\prime} \cap U_{1}, N^{\prime} \cap U_{1}\right) \rightarrow\left(U_{1}, M \cap U_{1}, N \cap U_{1}\right)
$$

which can be extended to an $f_{1} \in H(X)$ by $f_{1} \mid X \backslash U_{1}=$ id. Then $f_{1}\left(M^{\prime}\right) \cap U_{1}=$ $f_{1}\left(M^{\prime}\right) \cap f_{1}\left(U_{1}\right)=f_{1}\left(M^{\prime} \cap U_{1}\right)=M \cap U_{1}$. Similarly we have $f_{1}\left(N^{\prime}\right) \cap U_{1}=N \cap U_{1}$. Since $\left(f_{1}\left(M^{\prime}\right) \cap U_{2}, f_{1}\left(N^{\prime}\right) \cap U_{2}\right)$ is a (cap, f.d. cap)-pair for $U_{2}$, we have a homeomorphism

$$
h_{2}:\left(U_{2}, f_{1}\left(M^{\prime}\right) \cap U_{2}, f_{1}\left(N^{\prime}\right) \cap U_{2}\right) \rightarrow\left(U_{2}, M \cap U_{2}, N \cap U_{2}\right)
$$

which can be extended to an $f_{2} \in H(X)$ by $f_{2} \mid X \backslash U_{2}=$ id. Since $f_{2} f_{1}\left(M^{\prime}\right) \cap$ $U_{2}=M \cap U_{2}$ and

$$
\begin{aligned}
f_{2} f_{1}\left(M^{\prime}\right) \cap\left(U_{1} \backslash U_{2}\right) & =f_{2}\left(\left(f_{1}\left(M^{\prime}\right) \cap U_{1}\right) \backslash U_{2}\right) \\
& =f_{2}\left(\left(M \cap U_{1}\right) \backslash U_{2}\right)=M \cap\left(U_{1} \backslash U_{2}\right),
\end{aligned}
$$

$f_{2} f_{1}\left(M^{\prime}\right) \cap\left(U_{1} \cup U_{2}\right)=M \cap\left(U_{1} \cup U_{2}\right)$. Similarly $f_{2} f_{1}\left(N^{\prime}\right) \cap\left(U_{1} \cup U_{2}\right)=$ $N \cap\left(U_{1} \cup U_{2}\right)$. By induction, we can construct $f_{i} \in H(X), i \in \mathbf{N}$, such that $f_{i} \mid X \backslash U_{i}=\mathrm{id}$,

$$
f_{i} \circ \cdots \circ f_{1}\left(M^{\prime}\right) \cap\left(U_{1} \cup \cdots \cup U_{i}\right)=M \cap\left(U_{1} \cup \cdots \cup U_{i}\right)
$$


and

$$
f_{i} \circ \cdots \circ f_{1}\left(N^{\prime}\right) \cap\left(U_{1} \cup \cdots \cup U_{i}\right)=N \cap\left(U_{1} \cup \cdots \cup U_{i}\right)
$$

We also note that $f_{1} \circ \cdots \circ f_{1}\left(U_{1} \cup \cdots \cup U_{i}\right) U_{1} \cup \cdots \cup U_{i}$. Let $f \in H(X)$ be the limit of $\left\{f_{i} \circ \cdots \circ f_{1}\right\}_{i \in \mathbf{N}}$. Then $f\left(M^{\prime}\right)=M$ and $f\left(N^{\prime}\right)=N$. In fact, for any $x \in M^{\prime} \cap U_{i}$,

$$
f(x)=f_{n_{i}} \circ \cdots \circ f_{n_{1}}(x) \in f_{n_{1}} \circ \cdots \circ f_{n_{i}}\left(M^{\prime}\right) \cap\left(U_{n_{1}} \cup \cdots \cup U_{n_{i}}\right) \subset M
$$

and for $y=f(x) \in M \cap f\left(U_{i}\right)$,

$$
\begin{aligned}
y & =f_{n_{i}} \circ \cdots \circ f_{n_{1}}(x) \in M \cap f_{n_{i}} \circ \cdots \circ f_{n_{1}}\left(U_{n_{1}} \cup \cdots \cup U_{n_{i}}\right) \\
& =M \cap\left(U_{n_{1}} \cup \cdots \cup U_{n_{i}}\right) \\
& =f_{n_{i}} \circ \cdots \circ f_{n_{1}}\left(M^{\prime} \cap\left(U_{n_{1}} \cup \cdots \cup U_{n_{i}}\right)\right) \\
& \subset f\left(M^{\prime}\right) .
\end{aligned}
$$

Hence $f\left(M^{\prime}\right)=M$. Similarly $f\left(N^{\prime}\right) \subset N$. Therefore

$$
\left(X, M^{\prime}, N^{\prime}\right) \cong(X, M, N),
$$

which implies that $(M, N)$ is a (cap, f.d. cap)-pair for $X$.

We can apply Theorem 2.1 to verify that various triples mentioned in the Introduction are homeomorphic to $(Q, \Sigma, \sigma)$ or $(s, \Sigma, \sigma)$. Combining Theorem 2.1 with Lemma 1.1, we have the following

2.2. Classification Theorem. Let $(X, M, N)$ and $\left(X^{\prime}, M^{\prime}, N^{\prime}\right)$ be $(Q, \Sigma, \sigma)$ ( or $(s, \Sigma, \sigma)$-)manifold triples. Then $(X, M, N) \cong\left(X^{\prime}, M^{\prime}, N^{\prime}\right)$ if and only if $X \cong X^{\prime}$. Moreover each homeomorphism $f: X \rightarrow X^{\prime}$ is approximated by homeomorphisms $h: X \rightarrow X$ such that $h(M)=M^{\prime}$ and $h(N)=N^{\prime}$.

The following follows from the Stability Theorem for $Q$ - (or $s$-)manifolds [ AS ] and Theorem 2.2.

2.3. Stability Theorem. Let $(X, M, N)$ be a $(Q, \Sigma, \sigma)$ ( or $(s, \Sigma, \sigma)$-)manifold triple. Then the projection $p: X \times Q \rightarrow X($ or $p: X \times s \rightarrow X)$ is approximated by homeomorphisms $h: X \times Q \rightarrow X($ or $h: X \times s \rightarrow X)$ such that $h(M \times \Sigma)=M$ and $h(N \times \sigma)=N$.

By Lemma 1.3, Theorems 2.1 and 2.2, the Triangulation Theorem for $Q$ - (or $s$-)manifolds $\left[\mathrm{Ch}_{2}\right]$ yields the following.

2.4. Triangulation Theorem. For any $(Q, \Sigma, \sigma)$ - (or $(s, \Sigma, \sigma)$-)manifold triple $(X, M, N)$, there is a countable locally finite simplical complex $K$ such that

$$
(X, M, N) \cong(|K| \times Q,|K| \times \Sigma,|K| \times \sigma) \quad(\text { or }(|K| \times s,|K| \times \Sigma,|K| \times \sigma)) .
$$

Lemma 1.4, Theorems 2.1 and 2.2 and the Open Embedding Theorem for $s$-manifolds [ $\mathrm{He}$ ] yield the following. 
2.5. Open Embedding Theorem. For any $(s, \Sigma, \sigma)$-manifold $(X, M, N)$, there is an open embedding $g: X \rightarrow s$ such that $g(M)=g(X) \cap \Sigma$ and $g(N)=$ $g(X) \cap \sigma$.

In the $(s, \Sigma, \sigma)$-manifold case, we have the following version of Theorem 2.2 since two $s$-manifolds having the same homotopy type are homeomorphic [ $\mathrm{He}$ ].

2.6. Classification Theorem II. Let $(X, M, N)$ and $\left(X^{\prime}, M^{\prime}, N^{\prime}\right)$ be $(s, \Sigma, \sigma)$ manifold triple. Then $(X, M, N) \cong\left(X^{\prime}, M^{\prime}, N^{\prime}\right)$ if and only if $X \cong X^{\prime}$ or $M \cong M^{\prime}$ of $N \cong N^{\prime}$.

\section{3. $(\Sigma, \sigma)$-MANIFOLD PAIRS AND $\Sigma \times s$-MANIFOLDS}

In [BM], Bestvina and Mogilski gave a characterization of $\Sigma \times s$-manifolds and established the Stability, Triangulation, Open Embedding Theorems, etc. In particular, they proved that $\Sigma \times s \cong s_{f}^{\omega}$, where $s_{f}^{\omega}=\left\{\left(x_{i}\right) \in s^{\omega} \mid x_{i}=\right.$ 0 except for finitely many $i\}$. Note that

$$
\Sigma \times s \cong \sigma \times Q \times s \cong \sigma \times s .
$$

The following can be shown by using the characterization of $\Sigma \times s$ [BM, Corollary 6.3], but we give here a different proof using Theorems 2.1 and 2.2.

\subsection{Theorem. $\Sigma \backslash \sigma \cong \Sigma \times s$.}

Proof. Note $\left(I^{\omega}, I_{f}^{\omega}\right) \cong(Q, \sigma)$. It is easy to see that $\left(I_{f}^{\omega} \times Q, I_{f}^{\omega} \times \sigma\right)$ is a (cap, f.d. cap)-pair for $I^{\omega} \times Q$. By Theorems 2.1 and 2.2,

$$
\left(I^{\omega} \times Q, I_{f}^{\omega} \times Q, I_{f}^{\omega} \times \sigma\right) \cong(Q, \Sigma, \sigma) .
$$

Since $Q \backslash \sigma \cong s\left[\mathrm{An}_{3}\right.$, Theorem 5.3] (cf. [ $\mathrm{Ch}_{1}$, Corollary 8.3]), we have

$$
\Sigma \backslash \sigma \cong\left(I_{f}^{(\omega} \times Q\right) \backslash\left(I_{f}^{\omega} \times \sigma\right)=I_{f}^{\omega} \times(Q \backslash \sigma) \cong \sigma \times s \cong \Sigma \times s .
$$

We have the following relationship between $(\Sigma, \sigma)$-manifolds and $\Sigma \times s$ manifolds.

3.2. Corollary. (1) For any $(\Sigma, \sigma)$-manifold pair $(M, N), M \backslash N$ is a $\Sigma \times s$ manifold. (2) Each $\Sigma \times s$-manifold is homeomorphic to $M \backslash N$ for some $(\Sigma, \sigma)$ manifold pair $(M, N)$.

Proof. Clearly Theorem 3.1 implies (1), and (2) follows from the Triangulation Theorem for $\Sigma \times s$-manifolds [BM, Theorem 3.1 and Lemma 1.3].

We next establish the following lemma which is needed in the proof of Theorem 3.4.

3.3. Lemma. Let $(M, N)$ be a $(\Sigma, \sigma)$-manifold pair and $\left(M^{\prime}, n^{\prime}\right)$ a (cap, f.d. cap )-pair for an s-manifold. If $M \cong M^{\prime}$ then $(M, N) \cong\left(M^{\prime}, N^{\prime}\right)$.

Proof. We may assume $M=M^{\prime}$. There is a countable star-finite open cover $\mathscr{U}=\left\{U_{i}\right\}_{i \in \mathbf{N}}$ of $M$ and open embeddings $\phi_{i}: U_{i} \rightarrow \Sigma, i \in \mathbf{N}$, such that 
$\phi_{i}\left(U_{i} \cap N\right)=\phi_{i}\left(U_{i}\right) \cap \sigma$. By [ AHW, Theorem 2] there is a sequence $n_{1} \leq n_{2} \leq$ $\ldots$ in $\mathbf{N}$ such that $f_{i} \in H(M)$, satisfying $f_{1} \mid M \backslash U_{i}=$ id ; then $\left\{f_{i} \circ \cdots \circ f_{1}\right\}_{i \in \mathbf{N}}$ converges pointwise to $f \in H(M)$ with $f\left|U_{i}=f_{n_{i}} \circ \cdots \circ f_{n_{1}}\right| U_{i}$.

Let $\mathscr{V}$ be an open cover of $U_{1}$ such that $\mathrm{st}^{6} \mathscr{V}$ is normal relative to $M \backslash U_{1}$. For each $V \in \mathscr{V}$, let $V^{X}$ and $V^{s}$ be open sets in $X$ and $s$ respectively such that $V^{X} \cap M=V$ and $V^{s} \cap \Sigma=\phi_{1}(V)$. Let $\mathscr{V}^{X}=\left\{V^{X} \mid V \in \mathscr{V}\right\}$, $\mathscr{V}^{s}=\left\{V^{s} \mid V \in \mathscr{V}\right\}, U_{1}^{X}=\bigcup \mathscr{V}^{X}$ and $U_{1}^{s}=\bigcup \mathscr{V}^{s}$. Then $U_{1}^{X} \cap M=U_{1}$, $U_{1}^{X} \cap N^{\prime}=U_{1} \cap N^{\prime}, U_{1}^{s} \cap \Sigma=\phi_{1}\left(U_{1}\right)$ and $U_{1}^{s} \cap \sigma=\phi_{1}\left(U_{1} \cap N\right)$. By Lemma 1.4, $\left(U_{1}, U_{1} \cap N^{\prime}\right)$ and $\left(\phi_{1}\left(U_{1}\right), \phi_{1}\left(U_{1} \cap N\right)\right)$ are (cap, f.d. cap)-pair for $U_{1}^{X}$ and $U_{1}^{s}$ respectively. Since the inclusions $i: U_{1} \subset U_{1}^{X}$ and $j: \phi_{1}\left(U_{1}\right) \subset U_{1}^{s}$ are fine homotopy equivalences, there are maps $f: U_{1}^{X} \rightarrow U_{1}$ and $g: U_{1}^{s} \rightarrow \phi_{1}\left(U_{1}\right)$

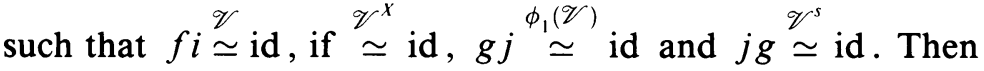

$$
\left(i \circ \phi_{1}^{-1} \circ g\right)\left(j \circ \phi_{1} \circ f\right) \stackrel{\mathscr{V}}{\simeq} i \circ f^{\mathscr{V}^{x}} \simeq \mathrm{id}
$$

and

$$
\left(j \circ \phi_{1} \circ f\right)\left(i \circ \phi_{1}^{-1} \circ g\right) \stackrel{\phi_{1}(\mathscr{V})}{\simeq} j \circ g \stackrel{\mathscr{V}^{s}}{\simeq} \mathrm{id} .
$$

Note that $\left(j \circ \phi_{1} \circ f\right)^{-1}\left(\mathscr{V}^{s}\right)=f^{-1}(\mathscr{V})$ and $\mathscr{V}^{X}<$ st $f^{-1}(\mathscr{V})$. Hence st $\mathscr{V}^{X}<$ $\mathbf{s t}^{2}\left(j \circ \phi_{1} \circ f\right)^{-1}\left(\mathscr{V}^{s}\right)=\left(j \circ \phi_{1} \circ f\right)^{-1}\left(\mathbf{s t}^{2} \mathscr{V}^{s}\right)$. Thus $j \circ \phi_{1} \circ f: U_{1}^{X} \rightarrow U_{1}^{s}$ is a $\mathrm{st}^{2} \mathscr{V}^{s}$-homotopy equivalence, which is $\mathrm{st}^{4} \mathscr{V}^{s}$-close to a homeomorphism by [ Fe, Theorem 3.4]. By Theorems 1.2 and 2.2, we have a homeomorphism

$$
h_{1}:\left(U_{1}^{X}, U_{1}, U_{1} \cap N^{\prime}\right) \rightarrow\left(U_{1}^{s}, \phi_{1}\left(U_{1}\right), \phi_{1}\left(U_{1} \cap N\right)\right)
$$

which is $\mathbf{s t}^{5} \mathscr{V}^{s}$-close to $j \circ \phi_{1} \circ f$. Since $h_{1} \mid U_{1}$ is st $^{5} \phi_{1}(\mathscr{V})$-close to $\phi_{1} f i$,

$$
\phi_{1}^{-1} h_{1} \mid U_{1}:\left(U_{1}, U_{1} \cap N^{\prime}\right) \rightarrow\left(U_{1}, U_{1} \cap N\right)
$$

is $\mathrm{st}^{5} \mathscr{V}$-close to $f i$, hence $\mathrm{st}^{5} \mathscr{V}$-close to id. Since $\mathrm{st}^{6} \mathscr{V}$ is normal relative to $M \backslash U_{1}, \phi_{1}^{-1} h_{1} \mid U_{1}$ extends to a $f_{1} \in H(M)$ with $f_{1} \mid M \backslash U_{1}=$ id and $f_{1}\left(N^{\prime}\right) \cap U_{1}=N \cap U_{1}$. By Corollary 1.6 and Theorem 1.2, $\left(X, M, f_{1}(N)\right)$ is an $(s, \Sigma, \sigma)$-manifold triple. By the same procedure, we have a $f_{2} \in H(M)$ with $f_{2} \mid M \backslash U_{2}=$ id and $f_{2} f_{1}(N) \cap U_{2}=N^{\prime} \cap U_{2}$. Similar to the implication (i) $\Rightarrow$ (ii) in Thoerem 2.1, $f_{2} f_{1}\left(N^{\prime}\right) \cap\left(U_{1} \cup U_{2}\right)=N \cap\left(U_{1} \cup U_{2}\right)$. By induction we obtain, for each $i \in \mathbf{N}$, an $f_{i} \in H(M)$, such that $f_{i} \mid M \backslash U_{i}=$ id and

$$
f_{i} \circ \cdots \circ f_{1}\left(N^{\prime}\right) \cap\left(U_{1} \cup \cdots \cup U_{i}\right)=N \cap\left(U_{1} \cup \cdots \cup U_{i}\right) .
$$

Let $f \in H(M)$ be the limit of $\left\{f_{i} \circ \cdots \circ f_{1}\right\}_{i \in \mathbf{N}}$. Then $f\left(N^{\prime}\right)=N$. Therefore $\left(M^{\prime}, N^{\prime \prime}\right) \cong(M, N)$.

Since each $\Sigma$-manifold $M$ is homeomorphic to $|K| \times \Sigma$ for some countable locally finite simplicial complex $K$, we have the theorem which followed from Lemmas 3.1 (using Theorem 2.1) and Lemma 3.3. 
3.4. Theorem. Each $(\Sigma, \sigma)$-manifold pair can be embedded as a (cap, f.d. cap)pair for an s-manifold and also for a Q-manifold.

Thus we have the $(\Sigma, \sigma)$-manifold pair versions for Theorems 2.2-2.6. We now give a characterization of $(\Sigma, \sigma)$-manifold pairs:

3.5. Theorem. Let $(M, N)$ be a pair of spaces. $(M, N)$ is a $(\Sigma, \sigma)$-manifold pair if and only if $M$ is an $A N R$ and $M=\bigcup_{n \in \mathbf{N}} M_{n}$, where $M_{1} \subset M_{2} \subset \ldots$ is a tower of compact $Q$-manifolds satisfying $(*)^{\prime}$ and $(* *)$.

Proof. The "only if" part is a direct consequence of Theorem 3.4 and Lemma 1.5. We need to show the "if" part. By [ CDM, Proposition 2.2] and [Mo], $M$ is a $\Sigma$-manifold. Since $M$ can be embedded as a cap set for an $s$-manifold [ $\left.\mathrm{Ch}_{1}\right],(M, N)$ is a $(\Sigma, \sigma)$-manifold pair by Lemma 1.5 and Theorem 2.1.

By Lemma 1.5, Theorems 2.1 and 3.5, we state another characterization of $(Q, \Sigma, \sigma)$ - (or $(s, \Sigma, \sigma)$-)manifold triples:

3.6. Theorem. Let $X$ be a $Q$ - (or s-)manifold and $N \subset M \subset X$. Then $(X, M, N)$ is a $(Q, \Sigma, \sigma)-($ or $(s, \Sigma, \sigma)$-) manifold triple if and only if $(M, N)$ is a $(\Sigma, \sigma)$-manifold pair and $M$ or $N$ is map-dense in $X$.

In other words, this theorem asserts that $(X, M, N)$ is a $(Q, \Sigma, \sigma)$ - (or $(s, \Sigma, \sigma)$-manifold triple if and only if $(X, M)$ is a $(Q, \Sigma)$ - (or $(s, g E)$-)manifold pair (or $(X, N)$ is a $(Q, \sigma)$ - (or $(s, \sigma)$-) manifold pair) and $(M, N)$ is a $(\Sigma, \sigma)$-manifold pair.

3.7. Problem. (i) If $M$ is a $\Sigma$-manifold and $N$ is a $\sigma$-manifold such that $N \subset M$ and $M \backslash N$ is a $\Sigma \times s$-manifold, then is $(M, N)$ a $(\Sigma, \sigma)$-manifold pair? (ii) If $M$ is a cap set and $N$ is an f.d. cap set for a $Q$ - (or $(s$-)manifold $X$ such that $N \subset M$ and $M \backslash N$ is a $\Sigma \times s$-manifold, then is $(X, M, N)$ a $(Q, \Sigma, \sigma)$ - (or $(s, \Sigma, \sigma)$-)manifold triple?

\section{REFERENCES}

[An $n_{1}$ R. D. Anderson, Hilbert space is homeomorphic to the countable infinite product of lines, Bull. Amer. Math. Soc. 72 (1966), 515-519.

$\left[\mathrm{An}_{2}\right]$ _- Strongly negligible sets in Fréchet manifolds, Bull. Amer. Math. Soc. 75 (1969), 64-67.

$\left[\mathrm{An}_{3}\right]$ _. On sigma-compact subsets of infinite-dimensional spaces, unpublished manuscript.

[AC] R. D. Anderson and T. A. Chapman, Extending homeomorphisms to Hilbert cube manifolds, Pacific J. Math. 38 (1971), 281-293.

[AHW] R. D. Anderson, D. W. Henderson, and J. E. West, Negligible subsets of infinite-dimensional manifolds, Compositio Math. 21 (1969), 143-150.

[AM] R. D. Anderson and J. D. McCharen, On extending homeomorphisms to Fréchet manifolds, Proc. Amer. Math. Soc. 25 (1970), 283-289.

[AS] R. D. Anderson and R. M. Schori, Factors of infinite-dimensional manifolds, Trans. Amer. Math. Soc. 142 (1969), 315-330.

$\left[\mathrm{BP}_{1}\right] \quad \mathrm{C}$. Bessaga, and A. Pelczyński, The estimated extension theorem, homogeneous collections and skeletons, and their application to the topological classification of linear metric spaces and convex sets, Fund. Math. 69 (1970), 153-190. 
$\left[\mathrm{BP}_{2}\right]-\frac{1}{1970}$, Selected topics in infinite-dimensional topology, Monogr. Mat. 58, PWN, Warsaw, 1970.

[BM] M. Bestvina, and J. Mogilski, Characterizing certain incomplete infinite-dimensional absolute retracts, Michigan Math. J. 33 (1986), 291-313.

[Ch $\left.{ }_{1}\right]$ T. A. Chapman, Dense sigma-compact subsets of infinite-dimensional manifolds, Trans. Amer. Math. Soc. 154 (1971), 399-426.

$\left[\mathrm{Ch}_{2}\right]$ - Lectures on Hilbert cube manifolds, CBMS Regional Conf. Ser. in Math., no. 28, Amer. Math. Soc., Providence, R.I., 1976.

[Cu] D. W. Curtis, Boundary sets in Hilbert cube, Topology Appl. 20 (1985), 201-221.

[CDM] D. Curtis, T. Dobrowolski, and J. Mogilski, Some applications of the topological characterizations of the sigma-compact spaces $l_{f}^{2}$ and $\Sigma$, Trans. Amer. Math. Soc. 284 (1984), 837-846.

[En] R. Engelking, General topology, Monogr. Mat., 60, PWN, Warsaw, 1977.

[Fe] S. Ferry, The homeomorphism group of a subcompact Hilbert cube manifold is an ANR, Ann. of Math. (2) 106 (1977), 101-119.

[Ge] R. Geoghegan, On spaces of homeomorphisms, embeddings, and functions. II. The piecewise linear case, Proc. London Math. Soc. (3) 27 (1973), 463-483.

[He] D. W. Henderson, Infinite dimensional manifolds are open subsets of Hilbert space, Topology 9 (1970), 25-33.

[Mo] J. Mogilski, Characterizing the topology of infinite-dimensional $\sigma$-compact manifolds, Proc. Amer. Math. Soc. 92 (1984), 111-118.

[Sa $\left.\mathrm{Sa}_{1}\right]$ K. Sakai, On topologies of triangulated infinite-dimensional manifolds, J. Math. Soc. Japan 39 (1987), 287-300.

$\left[\mathrm{Sa}_{2}\right] \ldots, \mathrm{A}$ Q-manifold local-compactification of metric combinatorial $\infty$-manifold, Proc. Amer. Math. Soc. 100 (1987).

$\left[\mathrm{Sa}_{3}\right] \quad$ - The space of Lipschitz maps from a compactum to a polyhedron, in preparation.

[SW] K. Sakai and R. Y. Wong, The space of Lipschitz maps from a compactum to a locally convex set, Topology Appl. (submitted).

[We] J. E. West, Infinite products which are Hilbert cubes, Trans. Amer. Math. Soc. 150 (1970), $1-25$.

Institute of Mathematics, University of Tsukuba, Sakura-mura, Ibaraki, 305 Japan

Department of Mathematics, University of California, Santa Barbara, California 93106 